Perspectiva de Familia (Perspect. fam.) ISSN 2415-5187, año 2016, vol. 1, pp. 11-22

\title{
Percepción de los estilos de crianza y felicidad en adolescentes y jóvenes de Lima Metropolitana
}

Perceived Parenting Styles and Happiness in Teenagers and Young Adults from Lima Metropolitan

\section{Resumen}

El presente estudio tiene por objetivo determinar la relación entre la percepción de estilos de crianza y la felicidad en una muestra de 102 adolescentes y jóvenes de Lima Metropolitana, 64 mujeres y 38 varones con edades entre 17 y 28 años. El diseño de la investigación es correlacional y predictivo. Se emplearon como instrumentos de recojo de información la Escala de Crianza Percibida «Mis recuerdos de la infancia» (Forma Corta) y la Escala de Felicidad de Lima. Los resultados permiten concluir que existen correlaciones significativas entre las dimensiones de la percepción de estilos de crianza y felicidad en la muestra estudiada. Para determinar cuál o cuáles de las dimensiones de la percepción de estilos de crianza predicen mejor la felicidad, se ha realizado un análisis de regresión múltiple, identificando que la variable Rechazo-padre y Calidez Emocional-Madre explican el 23 \% de la varianza total de la variable Felicidad.

Palabras clave: Análisis predictivo, estilos de crianza, felicidad, psicología positiva.

\begin{abstract}
The aim of the current study is to find the correlation between perceived parenting styles and happiness in a sample of 102 teenagers and young adults from Lima Metropolitan, 64 women and 38 men ranging in age from 17 to 28 years. The design of this study is correlational and predictive. The instruments used to collect information were the Scale
\end{abstract}


of Perceived Parenting "My childhood memories" (EMBU-Short Form), and the Happiness Scale of Lima. The results indicate that there are significant correlations between the dimensions of perceived parenting styles and happiness in the sample studied. A multiple regression analysis has been done to determine which of the dimensions of perceived parenting styles is the best predictor of happiness, the analysis reveal that the variable father's rejection and mother's emotional warmth explained $23 \%$ of the total variance in Happiness.

Key words: Predictive analysis, parenting styles, happiness, positive psychology.

La psicología positiva, impulsada por Seligman y Csikszentmihalyi (200o), es un enfoque orientado a promover una mejor calidad de vida, a partir del estudio de un conjunto de rasgos psicológicos positivos. Ante ello, esta corriente aborda científicamente numerosos temas de estudio, como la felicidad, la gratitud, la esperanza, entre otros (Alarcón, 2009), siendo en la actualidad la felicidad y la percepción de los estilos de crianza — variables que se estudiarán en el presente trabajo- fenómenos considerados importantes para la adaptación del individuo y como predictores del bienestar y la salud (Seligman, 2011).

La felicidad, como tema central de la psicología positiva (Alarcón, 2003; Caycho, 2010), ha sido definida de distintas maneras. En primer lugar, puede ser entendida como bienestar subjetivo (Diener, 200o), lo que trae como consecuencia que ambos términos, felicidad y bienestar subjetivo, sean utilizados en muchas oportunidades como sinónimos (Alarcón \& Caycho, 2013). En segundo lugar, a partir de la teoría de la experiencia óptima (Csikszentmihalyi, 1997), la felicidad es entendida como un estado de satisfacción producido por el logro de alcanzar algo que deseamos.

A partir de una serie de estudios en el contexto peruano, Alarcón (2009) señala que la felicidad es un estado afectivo de satisfacción, experimentado subjetivamente por un individuo como resultado del logro de obtener un bien u objeto deseado, los cuales pueden ser de diversa naturaleza. En base a esta última definición, Alarcón (2006) indica que la felicidad está compuesta por una ausencia de estados depresivos y pesimismo, que él denomina la dimensión de ausencia de sufrimiento profundo; la satisfacción debido al logro de las metas propuestas y la tranquilidad emocional a partir de la orientación hacia metas consideradas como importantes (denominadas satisfacción con la vida y realización personal, respectivamente), y, finalmente, el optimismo y la alegría (denominado alegría de vivir).

En relación a los estilos de crianza, es escasa la literatura que analiza su relación con el desarrollo de características positivas y negativas en los individuos (Rojas, 2015). Podemos definir la percepción de los estilos de crianza como un conjunto de actitudes, sentimientos y comportamientos de los padres hacia los hijos tal como son percibidas por estos (Darling \& Steinberg, 1993) y que se encuentran relacionadas con su desarrollo 
y bienestar (Broderick \& Blewitt, 2003). Richaud de Minzi (2005), a partir de un modelo cognitivo, señala que la percepción que tienen los hijos acerca del tipo de relación con sus padres, en comparación al comportamiento de los mismos, es la variable que más influye en el comportamiento de los adolescentes.

En relación a lo anterior, algunos autores (Baumrind, 1966) definen los estilos de crianza en base al control y exigencia ejercido por los padres y el grado en que responden a las necesidades de sus hijos (Richaud et al., 2013), lo que se encuentra asociado como la individualidad, la auto-regulación y la autoafirmación de los hijos (Broderick \& Blewitt, 2003), cuya combinación genera tres estilos de crianza diferentes: primero, el autoritario, caracterizado por una alta exigencia y baja respuesta a las necesidades de sus hijos, empleando, en muchos casos, el castigo físico y verbal. En segundo lugar, el estilo permisivo, implica una baja exigencia y alta receptividad a las necesidades, lo que puede estar relacionado con resultados positivos en el bienestar infantil. Finalmente, se plantea la existencia del estilo autorizado, caracterizado por una moderada exigencia y receptividad. A la clasificación anterior se agrega un cuarto estilo, denominado negligente, caracterizado como una falta de receptividad y exigencia (Richaud et al., 2013). En 1965, Schaefer propone un modelo tridimensional de los estilos de crianza parental constituido por la aceptación frente al rechazo, autonomía psicológica frente al control psicológico y el control firme frente a control laxo (Caycho, 2016).

Numerosos estudios (Domenech, Donovick \& Crowley, 2009; Sandler, Wolchick, MacKinnon, Ayers \& Roosa, 1997; Skinner \& Welborn, 1994) señalan que la percepción de apoyo de los padres por parte de los hijos se asocia con elevados niveles de autoestima, integración social, percepción de control y efectividad en el afrontamiento, habilidad de los adolescentes para afrontar los problemas, comprensión de las conductas y actitudes de los padres.

Respecto a la relación entre felicidad y estilos de crianza, son pocas las investigaciones que la estudian de manera directa (Rojas, 2015). Estos pocos estudios señalan que existe una relación entre los estilos de crianza y felicidad, presentando mayor asociación con estilos democráticos y de control parental (Furnham \& Cheng, 20oo; Petito \& Cummins, 200o; Suldo \& Huebner, 2004; Engin et al., 2013). En este sentido, el estilo autoritario de la madre es la variable que mejor predice el estado de felicidad de sus hijos (Furnham \& Cheng, 2000). En relación al efecto de los estilos de crianza de ambos padres, Raboteg-Saric y Sakic (2014) señalan que los estilos de crianza permisivo se encuentran asociados con elevados niveles de felicidad, en comparación con otros estilos.

En consecuencia, el presente trabajo se propone, en primer lugar, determinar si existen relaciones entre las variables felicidad y estilos de crianza. Finalmente, como segundo objetivo, está el determinar si la percepción de los estilos de crianza predice con mayor probabilidad la felicidad. 


\section{Método}

Se realizó un estudio ex post facto prospectivo (Montero \& León, 2007) donde no se manipularon las variables de estudio, más bien se analizaron con el objetivo de determinar la relación entre ellas.

\section{Participantes}

La presente investigación contó con la participación de 102 adolescentes y jóvenes universitarios (62,7 \% mujeres y $37.3 \%$ hombres) cuyas edades fluctúan entre 17 a 27 años, de las facultades de psicología, medicina, ciencias de la comunicación e ingeniería de cuatro universidades privadas de Lima Metropolitana, seleccionados en base al muestreo probabilístico. Se ha tomado en cuenta como criterios de exclusión que los participantes sean de nacionalidad extranjera, que tengan hijos, que sean menores de 17 o mayores de 27 años y que no se encuentren cursando estudios superiores.

\section{Instrumentos}

Ficha de datos sociodemográficos. Cuestionario diseñado para fines de esta investigación donde se recolecta información de los participantes acerca de: ciclo de estudios, edad, sexo, estado civil, grado de instrucción, trabajo, lugar de nacimiento, personas con las que convive, si tiene hijos y la carrera que estudian.

Escala de crianza percibida «Mis recuerdos de la infancia» (S-EMBU; Arrindel et al., 1999). La versión original fue desarrollada por Perris et al. (1980) con el objetivo de evaluar la crianza de los padres tal como es percibida por los hijos. Para efectos de la investigación, se emplea una versión abreviada (Arrindell, et al., 1999), compuesta por 23 ítems con cuatro alternativas de respuesta. La escala consta de tres dimensiones: la escala Rechazo (7 ítems), evalúa la presencia de hostilidad física o verbal, conductas punitivas, agresión y expectativas rígidas con respeto a los logros en el niño; la escala Calidez Emocional (6 ítems), evalúa las expresiones de aceptación, confianza, apoyo y consuelo ante la adversidad en función al punto de vista del evaluado; por último, la escala de Sobreprotección (9 ítems), que hace referencia a la excesiva preocupación por la seguridad del hijo. La escala cuenta con evidencias de adecuadas propiedades psicométricas de confiabilidad y validez convergente y discriminante (Arrindell \& Engebretsen, 200o; Parker, Tupling \& Brown, 1979). Un estudio previo en población peruana general (Merino, 2013) halló que la estructura dimensional fue satisfactoria. En el presente estudio la consistencia para Rechazo $\left(\alpha_{\text {padres }}=0.80\right.$, IC 95 \% = 0.71, 0.86; $\alpha_{\text {madres }}=0.75$, IC $\left.95 \%=0.65,0.82\right)$, Calidez Emocional $\left(\alpha_{\text {padres }}=0.84\right.$, IC $95 \%=0.77$, $0.88 ; \alpha_{\text {madres }}=0.87$, IC $\left.95 \%=0.81,91\right)$ y Sopreproteccion $\left(\alpha_{\text {padres }}=0.70\right.$; IC $95 \%=0.58$, $0.78 ; \alpha_{\text {madres }}=0.74$, IC $\left.95 \%=0.63,0.81\right)$ fueron adecuadas. 
Escala de Felicidad de Lima (EFL) de Alarcón (2006), compuesta por 27 ítems de tipo Likert, con cuatro alternativas de respuesta: totalmente de acuerdo, de acuerdo, ni de acuerdo ni desacuerdo, desacuerdo y totalmente en desacuerdo. Al extremo positivo se le otorgaron cinco puntos y al extremo negativo un punto. Los ítems fueron redactados en forma favorable o desfavorable a la felicidad, siendo distribuidos aleatoriamente. La EFL ha sido administrada a 709 personas, varones y mujeres con una edad promedio de 23.10 años. Evalúa cuatro dimensiones: Ausencia de sufrimiento profundo (11 ítems; $\alpha$ $=0.88$, IC $95 \%=0.82,0.91$ ), Satisfacción con la vida (6 ítems; $\alpha=0.79$, IC $95 \%=0.70$, o.85), Realización personal (6 ítems; $\alpha=0.76$, IC $95 \%=0.66,0.83$ ) y Alegría de vivir (4 ítems; $\alpha=0.72$, IC $95 \%=0.61,0.80$ ).

\section{Procedimiento}

Las pruebas fueron administradas juntas en forma colectiva a la muestra de clases. Antes de que los estudiantes dieran respuesta a los ítems se les pidió que leyeran cuidadosamente las instrucciones de cada prueba, recordándoles que no existe límite de tiempo para responder a ambas escalas. Los participantes completaron los datos referentes a edad, sexo, estado civil, situación laboral, conservando su anonimato en diligenciamiento de las escalas. Participaron de forma voluntaria y anónima, firmando el consentimiento informado que garantiza la confiabilidad de los datos suministrados. El estudio recibió la aprobación, previa a la aplicación, del comité de ética de la universidad correspondiente. En relación al análisis de datos, se aplicaron correlaciones paramétricas y análisis de regresión múltiple.

\section{Resultados}

\section{Correlación entre los tres factores de Estilos de Crianza y los cuatro factores de la Felicidad}

La Tabla 1 muestra las correlaciones entre las dimensiones de la percepción de los estilos de crianza y los cuatro factores de la felicidad. Se observa que existen correlaciones negativas y significativas entre el rechazo y sobreprotección de ambos padres y la felicidad, así como una correlación positiva y significativa entre esta última y la calidez emocional. Así, una mayor percepción de la calidez emocional de ambos padres, junto con un menor rechazo y sobreprotección, estaría asociada con mayores niveles de felicidad. Así mismo, se observa que la ausencia de sufrimiento se relaciona negativamente y de manera significativa con el rechazo de ambos padres y la sobreprotección del padre; mientras que se relaciona positiva y significativamente con la calidez emocional de ambos padres. 
Tabla 1.

Correlaciones de Pearson entre percepción de estilos de crianza y felicidad

\begin{tabular}{|c|c|c|c|c|c|}
\hline & Felicidad & $\begin{array}{l}\text { Ausencia de } \\
\text { sufrimiento }\end{array}$ & $\begin{array}{c}\text { Satisfacción con } \\
\text { la vida }\end{array}$ & $\begin{array}{c}\text { Realización } \\
\text { personal }\end{array}$ & $\begin{array}{l}\text { Alegría de } \\
\text { vivir }\end{array}$ \\
\hline \multicolumn{6}{|c|}{ Padre } \\
\hline Rechazo & $-0.332 * *$ & $-0.400 * *$ & $-0.222 *$ & -0.158 & -0.166 \\
\hline Calidez emocional & $0.367 * *$ & $0.300 * *$ & $0.350 * *$ & $0.252 *$ & $0.278 * *$ \\
\hline Sobreprotección & $-0.230 *$ & $-0.248^{*}$ & -0.148 & $-0.202 *$ & -0.85 \\
\hline \multicolumn{6}{|c|}{ Madre } \\
\hline Rechazo & $-0.343 * *$ & $-0.297 * *$ & $-0.310 * *$ & $-0.288 * *$ & $-0.226^{*}$ \\
\hline Calidez emocional & $0.347^{* *}$ & $0.332 * *$ & $0.356^{* *}$ & $0.250 *$ & $0.297 * *$ \\
\hline Sobreprotección & $-0.223 * *$ & -0.118 & $-0.237 *$ & $-0.327 * *$ & $-0-109$ \\
\hline
\end{tabular}

${ }^{*} p<0.05 ;{ }^{* *} p<0.01$

La misma Tabla 1 muestra correlaciones positivas y significativas entre la satisfacción con la vida y la calidez emocional del padre y la madre, así como una correlación negativa y significativa con el rechazo de ambos padres y la sobreprotección de la madre. Se observan, a su vez, correlaciones positivas y significativas entre calidez emocional del padre y la madre y la realización personal; así como también, correlaciones negativas e igualmente significativas entre esta última variable y la sobreprotección de ambos padres y el rechazo de la madre. Finalmente, la alegría por vivir se relaciona positiva y significativamente con la calidez emocional de ambos padres y negativamente con el rechazo de la madre.

\section{Influencia de las dimensiones de la percepción de los estilos de crianza sobre la felicidad}

Para determinar cuál o cuáles de las dimensiones de la percepción de los estilos parentales (rechazo, calidez emocional, sobreprotección) en ambos padres (padre y madre) influyen en la percepción de felicidad, controlando la variabilidad de las otras variables, se realizó un análisis de regresión múltiple, empleando el método de pasos sucesivos. Se introdujeron las seis variables mencionadas, siendo cuatro de ellas eliminadas, quedando calidez emocional-madre y rechazo-padre como las variables explicativas que más variabilidad produjeron.

Los resultados arrojaron un coeficiente de determinación $R^{2}=.230$ (error estándar $=$ 17.427) que indica que las variables calidez emocional-madre y rechazo-padre explican el 23 \% de la varianza total de la variable criterio Felicidad. Un valor mayor del coeficiente de determinación múltiple indica un mayor poder explicativo de la ecuación de regresión y, por lo tanto, mayor poder de explicación sobre la variable dependiente. La $R^{2}$ corregida, más baja, explica el $21.4 \%$, y es estadísticamente significativa, $F(2,79)$ 
$=14.749, p<0.001)$, señala la existencia de una relación lineal significativa entre las variables explicativas y la variable felicidad.

Tabla 2.

Coeficientes de regresión múltiple B (no estandarizados), $\beta$ (estandarizados) y test $t$

\begin{tabular}{|l|c|c|c|c|c|}
\hline \multicolumn{1}{|c|}{ Modelo } & B & E.E. & B & $t$ & Sig. \\
\hline 1 (Constante) & 88.127 & 9.614 & & 9.167 & .000 \\
\hline Rechazo-padre & -1.626 & .463 & -.316 & -3.514 & .001 \\
\hline Calidez emocional-madre & 1.310 & .385 & .306 & 3.403 & .001 \\
\hline
\end{tabular}

a Variable dependiente: Felicidad

Los coeficientes de regresión no estandarizados (B), coeficientes de regresión estandarizados $(\beta)$ y los estadísticos relacionados con las variables predictoras se muestran en la Tabla 2. Los valores $t$ de los coeficientes de regresión beta de las variables predictoras son altamente significativos $(p<0.01)$, indicando que difieren de cero. Este resultado permite concluir que ambas variables (rechazo-padre y calidez emocional-madre) predicen significativamente la variable criterio Felicidad.

\section{Discusión}

Si bien, a nivel internacional, algunos estudios sugieren que el rechazo, distanciamiento, hostilidad y sobreprotección se relacionan con los procesos de adaptación y regulación emocional (Van Huisstede, 2013), en nuestro medio aún son escasas las investigaciones al respecto. A partir de esto, el presente estudio brinda una aproximación acerca de las relaciones entre la percepción de estilos de crianza y la felicidad en una muestra de jóvenes universitarios de Lima Metropolitana. Al analizar la relación entre la percepción de los estilos de crianza y felicidad, los resultados indican una asociación significativa entre las dimensiones de ambas variables.

Se observan correlaciones positivas y significativas entre calidez emocional del padre y de la madre con la felicidad, y correlaciones negativas significativas entre la dimensión rechazo-padre y felicidad; sobreprotección-padre y felicidad; rechazo-madre y felicidad y sobreprotección-madre y felicidad, lo cual concuerda con una serie de estudios que señalan que el bienestar subjetivo y satisfacción con la vida se relacionan de manera positiva con estilos de crianza democrático, y de manera negativa con los estilos autoritarios y sobreprotectores (Engin et al., 2013; Petito \& Cummins, 200o; Suldo \& Huebner, 2004). Este resultado puede ser explicado por la mediación, directa o indirecta, de variables afectivas y emocionales como la autoestima (Furnham \& Cheng, 2000). 
Así mismo, la ausencia de sufrimiento profundo se correlaciona positivamente con calidez emocional y negativamente con rechazo, lo cual concuerda con estudios (Carrobles \& Gámez ,2012; Van Huisstede, 2013) que señalan que pocas demostraciones de cariño y afecto, así como una falta o exceso de control, se asocian con frecuencia con una mayor ineficiencia para la regulación de las emociones, la cual es considerada como factor de protección frente al sufrimiento profundo y promotor de felicidad.

En relación a la dimensión satisfacción con la vida, se observan relaciones positivas con la calidez de ambos padres, y relaciones negativas con comportamientos de rechazo del padre y de la madre, resultado concordante con lo mencionado por Carrobles y Gámez (2012), para quienes estilos parentales caracterizados por la presencia de calidez emocional y control, así como ausencia de rechazo y sobreprotección, contribuyen al desarrollo de la autonomía y esquemas cognitivos adaptativos, consideradas como variables importantes para una evaluación positiva de la vida. En lo que respecta a las dimensiones de realización personal y alegría de vivir, ambos muestran correlaciones negativas con el rechazo y positivas con calidez emocional. Estudios (Carrobles \& Gámez, 2012; Macoby \& Martin, 1983) señalan que adecuados niveles de calidez emocional, ausencia de rechazo y sobreprotección por parte de los padres favorece el desarrollo de la estabilidad emocional y el vivir plenamente, características implicadas en el logro de la realización personal y alegría de vivir (Alarcón, 2009).

El análisis predictivo realizado permite observar que el estilo de crianza de rechazo por parte del padre y la calidez emocional de la madre, predicen significativamente la variable felicidad. Este resultado coincide con Cheng y Furnham (2004) y Holden y Miller (1999), quienes señalan que es importante distinguir dos dimensiones en la calidez de la relación entre padres e hijos: primero, entre aquellos padres que mantienen una relación cálida, de respeto y aceptación, y, segundo, padres cuya relación con los hijos es indiferente y de rechazo.

Los resultados correlacionales y predictivos sugieren que variables familiares, expresadas en este estudio a partir de la relación entre padres e hijos, cumplen un papel importante en el desarrollo de conductas positivas en la adultez temprana (Shaw, Krause, Chatters, Connell \& Ingersoll, 2004).

Los resultados de esta investigación deben ser considerados a luz de las siguientes limitaciones. En primer lugar, en este trabajo solo se consideró la participación del constructo percepción de los estilos de crianza, dejando de lado otros constructos familiares importantes al momento de predecir la felicidad, como, por ejemplo, la funcionalidad familiar. En segundo lugar, al ser la muestra extraída de una población universitaria limeña mediante un muestreo por conveniencia, los resultados tienen limitada capacidad de generalización al resto del país, dada las diferencias culturales y estilo de vida presentes en costa, sierra y selva. Finalmente, en tercer lugar, es necesario 
contar con una mayor cantidad de participantes de diferentes sectores sociales en próximas investigaciones con el fin de poner a prueba el modelo de regresión.

La importancia de este estudio radica en que nos brinda información pertinente tanto para la práctica clínica como para la implementación de planes de intervención y prevención relacionadas a la salud y el bienestar.

\section{Referencias}

Alarcón R. (2003). Hallazgos y reflexiones sobre la psicología de la felicidad, Teoría e Investigación en Psicología, 11, 159-176.

Alarcón, R. (2006). Desarrollo de una escala factorial para medir la felicidad. Revista Interamericana de Psicología, 1(4), 99-106.

Alarcón, R. (2009). Psicología de la felicidad. Introducción a la psicología positiva. Lima: Universidad Ricardo Palma.

Alarcón, R. (2011). Psicología Contemporánea. Ensayos. Lima: Editorial Universitaria.

Alarcón, R., \& Caycho, T. (2015). Relaciones entre gratitud y felicidad en estudiantes universitarios de Lima Metropolitana. Psychologia: avances de la disciplina, 9(1), 59-69.

Arrindell, W. A., Sanavio, E., Aguilar, G., Sica, C., Hatzichristou, C., Eisemann, M., Recinos, L. A., Gaszner, P., Peter, M., Bat-tagliese, G., Kállai, J., \& van der Ende, J. (1999). The development of a short form of the EMBU: Its appraisal with students in Greece, Guatemala, Hungary and Italy. Personality and Individual Differences, 27(4), 613-628. doi:10.1016/So191-8869(98)oo192-5

Arrindell, W. A., \& Engebretsen, A. A. (2000). Convergent validity of the Short-EMBU and the Parental Bonding Instrument (PBI): Dutch findings. Clinical Psychology and Psychotherapy, 7(4), 262-266. doi: 10.1002/1099-0879(200010) $7: 4<262:: A I D-C$ PP257>3.0.CO;2-9

Baumrind, D. (1966). The Discipline controversy revisited. Family Relations, 45(4), 405-414.

Broderick, P. C., \& Blewitt, P. (2003). The life span: Human development for helping professionals. Upper Saddle River, NJ: Pearson Education.

Carrobles, J., \& Gámez, M. (2012). Manual del psicólogo de familia: un nuevo perfil profesional. Madrid: Pirámide.

Caycho, T. (2010).Variables psicológicas asociadas con la felicidad en centros peri-urbanos y urbanos marginales de Lima. Scientia UCV, 2(1) 61-68.

Caycho, T. (2016). Relación con los padres y estrategias de afrontamiento en adolescentes de Lima. Propósitos y Representaciones, 4(1), 11-59. doi: 10.20511/pyr2016.v4n1.86 
Cheng, H., \& Furnham, A. (2004). Perceived parental rearing style, self-esteem and self-criticism as predictors of happiness, Journal of Happiness Studies 5(1), 1-21. doi: 10.1023/B:JOHS.0oooo21704.35267.05

Csikszentmihalyi, M. (1997). Fluir (flow). Una psicología de la felicidad. Barcelona: Editorial Kairós.

Darling, N. \& Steinberg, L. (1993). Parenting style as context: An integrative model. Psychological Bulletin, 113(3), 487-496. doi: 10.1037/0033-2909.113.3.487

Diener, E. (200o). Subjective well-being: The science of happiness and proposal for a national index. American Psychologist, 55(1), 34-43. doi: 10.1037/0oo3-066X.55.1.34

Domenech, M., Donovick, R., \& Crowley, S. (2009). Parenting styles in a cultural context: Observations of "protective parenting" in first generation latinos. Family Process, 48(2), 1-18. doi: 10.1111/j.1545-5300.2009.01277.x

Dwairy, M. (2007). Parental inconsistency versus parental authoritarianism: association with symptoms of psychological disorders. Journal of Youth and Adolescence, doi 10.1007/s10964-007-9169-3

Dwairy, M., \& Dor, A. (2009). Parenting and psychological adjustment of adolescent immigrants in Israel. Journal of Family Psychology, 23(3), 416-425. doi: 10.1037/ aoo15830

Engin, M., Deniz, L., Karakus, Ö., Zeliha, T., Jale, E., Zümra, Ö., \& Erdal, H. (2013). Parental Attitude Perceived by University Students as Predictors of Subjective Well-Being and Life Satisfaction. Scientific Research, 3(4), 169-173. doi: 10.4236/ psych.2013.43025

Furnham, A., \& Cheng, H. (200o). Perceived parental behaviour, self-esteem and happiness. Social Psychiatry and Psychiatric Epidemiology, 35(10), 463-470. doi: 10.1007/s001270050265

Holden, G. W., \& Miller, P. C. (1999). Enduring and different: A meta-analysis of the similarity in parents' child rearing. Psychological Bulletin, 125(2), 223. doi: 10.1037/0033-2909.125.2.223

Maccoby, E., \& Martin, J. (1983). Socialization in the context of the family: Parent-child interaction. En P. H. Mussen (Series Ed.) \& E. M. Hetherington (Vol. Ed.), Handbook ofChild Psychology, 4. Socialization, personality, and social development (4.th ed., pp. 1-101). New York: Wiley.

Merino, C., \& Arndt, S. (2013). Análisis factorial confirmatorio de la Escala de Estilos de Crianza de Steinberg: validez preliminar de constructo. Revista de Psicología, $22(2), 189-214$.

Montero, I., \& León, O. G. (2007). Guía para nombrar los estudios de investigación en Psicología. International Journal of Clinical and Health Psychology, 7(3), 847-862.

Parker, G., Tupling, H., \& Brown, L. B. (1979). A parental bonding instrument. British Journal of Medical Psychology, 52(1), 1-10. doi: 10.1111/j.2044-8341.1979.tbo2487.x 
Perris, C., Jacobsson, L., Lindström, H., Von Knorring, L., \& Perris, H. (1980). Development of a new inventory for assess-ing memories of parental rearing behaviour. Acta Psychiatrica Scandinavica, 61(4), 265-274. doi: 10.1111/j.160o0447.1980.tboo581.X

Petito, F., \& Cummins, R. A. (200o). Quality of life in adolescence: the role of perceived control, parenting style, and social support. Behaviour Change, 17(3), 196-207. doi: 10.1375/bech.17.3.196

Raboteg-Saric, Z., \& Sakic, M. (2014). Relations of parenting styles and friendship quality to self-esteem, life satisfaction and happiness in adolescents. Applied Research In Quality of Life, 9(3), 749-765. doi: 10.1007/s11482-013-9268-o

Radziszewska, B., Richardson, J. L., Dent, C. W., \& Play, B. R. (1996). Parenting style and adolescent suppressive symptoms, smoking, and academic achievement: Ethnic, gender, and SES differences. Journal of Behavioral Medicine, 19, 289-305. doi: 10.1007/BFo1857770

Richaud, M. C. (2005). Versión abreviada del Inventario de la Percepción de los Hijos acerca de las Relaciones con sus Padres para Adolescentes. Psicodiagnosticar, 15, 99-106.

Richaud, M. C., Mestre, M. V., Lemos, V., Tur, A., Ghiglione, M., \& Samper, P. (2013). La influencia de la cultura en los estilos parentales en contextos de vulnerabilidad social. Avances en Psicología Latinoamericana, 31(2), 419-431.

Rojas, M. (2015). Felicidad y estilos de crianza parental. Documento de Trabajo 016/2015. México D. F.: Centro de Estudios Espinosa Yglesias.

Sandler, I. N., Wolchick, S. A., MacKinnon, D., Ayers, T. S., \& Roosa, M. W. (1997). Developing linkages between theory and intervention in stress and coping processes. En S. A. Wolchick \& I. N. Sandler (Eds.), Handbook of Children's Coping. Linking Theory and Intervention (pp. 3-40). Nueva York: Plenum Press.

Schaefer, E. S. (1965). Children's reports of parental behavior: An Inventory, Child Development, 38(2), 413-424.

Seligman, M. E. P., \& Csikszentmihalyi, M. (200o). Positive psychology: an introduction. American Psychologist, 55, 5-14.

Seligman M. E. P. (2004). Aprende Optimismo. Barcelona: De bolsillo.

Shaw, B., Krause, N., Chatters, C., Connell, C. M., \& Ingersoll, B. (2004). Emotional support from parents early in life, aging, and Health. Psychology and Aging, 19(1), 4-12. doi: 10.1037/o882-7974.19.1.4

Skinner, E. A., \& Welborn, J. G. (1994). Coping during childhood and adolescence: A motivational perspective. En R. Lerner, D. Featherman \& M. Perlmutter (Eds.), Lifespan Development and Behavior, (vol. 12, pp. 91-123). Hillsdale, NJ: Lawrence Earlbaum Associates. 
Suldo, S. M., \& Huebner, E. S. (2004). The role of life satisfaction in the relationship between authoritative parenting dimensions and adolescent problem behavior. Quality-of-Life Research on Children and Adolescents, 66, 165-195. doi:10.1007/9781-4020-2312-5_9

Van Huisstede, L. (2013). Perceived Parenting, Emotion Regulation, and Adult Depression. (Tesis de Maestría inédita). Arizona State University, Arizona, USA. 